\title{
Splice variants of androgen receptor and prostate cancer
}

\author{
Orazio Caffo, Francesca Maines, Antonello Veccia, Stefania Kinspergher, \\ Enzo Galligioni
}

Medical Oncology Department, Santa Chiara Hospital, Trento, Italy

\begin{abstract}
Over the last ten years, two new-generation hormonal drugs and two chemotherapeutic agents have been approved for the treatment of metastatic castration-resistant prostate cancer. Unfortunately, some patients have primary resistance to them and the others eventually develop secondary resistance. It has recently been suggested that the presence of androgen receptor splice variants plays a leading role in the primary and secondary resistance to the new hormonal drugs, whereas their presence seem to have only a partial effect on the activity of the chemotherapeutic agents. The aim of this paper is to review the published data concerning the role of androgen receptor splice variants in prostate cancer biology, and their potential use as biomarkers when making therapeutic decisions.
\end{abstract}

\section{Introduction}

Our knowledge of the biology of particularly advanced disease prostate cancer (PC) has significantly improved over the years. It is now known that the development, growth and maturation of the prostate are regulated by androgens, which act by modulating androgen receptors (ARs), ${ }^{1}$ but androgens also play a central role in the development of PC. It was as long ago as 1941 that Huggins and

Correspondence: Orazio Caffo, Medical Oncology Department, Santa Chiara Hospital, Largo Medaglie d'Oro, 38100 Trento, Italy.

Tel.: +39.0461 .902478 - Fax: +39.0461 .903364 .

E-mail orazio.caffo@apss.tn.it

Key words: Prostate cancer; splice variants; enzalutamide; abiraterone; cabazitaxel.

Contributions: the authors contributed equally.

Conflict of interest: OC received honoraria from Sanofi Aventis, Janssen, Bayer and Astellas. The other authors do not have conflict of interest to be declared.

Received for publication: 28 March 2016.

Accepted for publication: 13 April 2016.

This work is licensed under a Creative Commons Attribution NonCommercial 4.0 License (CC BY-NC 4.0).

(C) Copyright O. Caffo et al., 2016

Licensee PAGEPress, Italy

Oncology Reviews 2016; 10:297

doi:10.4081/oncol.2016.297
Hodges first demonstrated that a castration-induced reduction in testosterone made it possible to control metastatic $\mathrm{PC},{ }^{2}$ which is still initially treated by means of androgen deprivation therapy. ${ }^{3}$ Unfortunately, most of the patients who have undergone androgen deprivation therapy eventually becomes resistant and progress ${ }^{4}$ a condition that was formerly defined as hormone refractory and thus suggested mechanisms of tumour growth that were unrelated to the AR axis. ${ }^{5}$ However, as this definition did not reflect the possibility that a patient may respond to other hormone-based strategies, it was considered more appropriate to change it to a castration-resistant state in which ARs continue to be expressed and AR signalling retains its central role in tumour growth. ${ }^{6}$ The ability of tumour cells to grow under conditions of testosterone castration and progression to castrationresistant PC (CRPC) is thus strictly related to the re-activation of the androgen/AR signalling axis, which may be due to various mechanisms: AR protein over-expression and $A R$ gene amplifications/mutations, ${ }^{7}$ the aberrant expression of co-activators and co-repressors, ${ }^{8}$ intracrine androgen synthesis, ${ }^{9}$ and alternative activation by means of tyrosine kinase-dependent signalling. ${ }^{10}$

The discovery that AR signalling plays a role in CRPC was the rationale for developing new-generation AR-targeting agents such as abiraterone acetate and enzalutamide, which have improved survival in both pre-treated and chemo-naïve metastatic CRPC (mCRPC) patients. ${ }^{11-14}$ However, some patients are primarily resistant to these agents, and all eventually develop acquired resistance. There is therefore increasing interest in the role of C-terminal truncated AR variants (AR-Vs) as biomarkers of the activity of new-generation AR-targeting agents and taxanes in metastatic CRPC. ${ }^{15,16}$

The aim of this paper is to review the available evidence concerning the role of AR splice variants in the development of PCs, and their value as biomarkers that can help when making decisions about the treatment of patients with metastatic CRPC.

\section{Androgen receptors variants structure and detection}

ARs are ligand-activated nuclear transcription factors ${ }^{17}$ encoded by a specific gene located on the X-chromosome at position Xq11-12. Fulllength ARs (AR-FL) contains four functional domains: an N-terminal domain (NTD), a central DNA-binding domain (DBD), a short hinge region, and a C-terminal ligand-binding domain (LBD). ${ }^{18}$

The interaction of androgen with the LBD leads to a series of sequential conformational changes in the receptor, which is flexible in the hinge region that not only acts as a link between the DBD and LBD, but also contains the nuclear localization signal (NLS) that binds the importin- $\alpha$ regulator of subsequent nuclear localization. ${ }^{19}$ ARs recognise and stably bind to androgen response elements (AREs), which are specific DNA elements whose activity is controlled by co-regulators and/or co-regulator complexes that influence nuclear AR targeting, 
ARE binding, and the spatial and temporal control of transcriptional activity. ${ }^{20}$

AR-Vs, which were first described in the CWR22 xenograft in which they were associated with progressive disease and resistance, ${ }^{21}$ are truncated AR species that mainly arise a result of the splicing of intronic sequences (i.e., cryptic exons). The most significant are those that lack the LBD, but retain the transcriptional activity mediated by NTD/DBD. ${ }^{22}$

Although the NLS disruption found in most AR-Vs could limit their nuclear translocation, currently unknown mechanisms lead to considerable constitutive localization in the nucleus, ${ }^{1}$ where they homo- and heterodimerise with each other or with AR-FLs. ${ }^{23}$ The heterodimerisation of AR-Vs and AR-FLs has been observed under conditions of low ligand concentrations, whereas AR-Vs may enhance AR-FL activity in the presence of high androgen levels. ${ }^{24,25}$ Moreover, the absence of the NLS hinge region leads to the escape of AR-V degradation mediated by E3 ubiquitin ligase adaptor speckle-type $\mathrm{POZ}$ protein. ${ }^{26}$ It is worth noting that AR co-regulators may also bind to and regulate AR-Vs, ${ }^{27-29}$ and that the target genes of AR-Vs and AR-FLs are very similar. ${ }^{30,31}$

AR-Vs are mainly generated by somatic rearrangements in the AR locus $^{32}$ or dynamic de-regulated AR splicing during hormonal manipulations. $^{33}$

\section{Androgen receptors variants detection}

It is difficult to assess the role of AR-Vs in the resistance and development of PC progression because our ability to detect their expression in biological samples is limited. Some preliminary studies of the relationship between AR-Vs and steroidogenic enzyme levels were based on AR-V mRNA expression, ${ }^{34}$ but the availability of specific antibodies suitable for tissue staining would be more sensitive and provide a more realistic picture: unfortunately, the only AR-V for which a specific antibody has so far been developed is AR-V7. ${ }^{27,35,36} \mathrm{An}$ alternative possibility is the concomitant use of two antibodies targeting either the NH2- or COOH-termini. ${ }^{37}$

Antonarakis et al. hypothesised that detecting AR-V7 RNA in circulating tumour cells taken from men with mCRPC may be helpful in identifying those with primary resistance to hormonal therapies and facilitate the selection of alternative treatments (e.g., chemotherapies). They detected circulating tumor cells (CTC) by means of commercially available non-quantitative platforms that could only determine their presence or absence, and used commercial kits for the purposes of isolation and enrichment. After capturing CTCs in blood samples taken from patients with mCRPC, mRNA expression was quantitatively analysed using reverse-transcriptase polymerase chain reaction primers to determine the ratio of AR-V and AR-FL transcripts. According to the CTC detection kit instructions, laboratory processing had to be carried out as early as possible and, in any case within two hours of collection, and the blood tubes had to be delivered to the laboratory on ice. The patients had AR-V7 positivity rates ranging from $19 \%$ to $39 \%,{ }^{15}$ but this is much lower than the $70 \%$ positivity reported by Hu et al. ${ }^{38}$ thus suggesting the need for a standardised and validated method.

\section{The role of androgen receptors variants}

Seventeen AR-Vs have been fully characterised so far, ${ }^{39}$ but nothing is known about the clinical significance of most of them.

Although the development of AR-Vs is usually considered as marking the transition from a less aggressive hormone-sensitive disease to a more aggressive castration-resistant condition, AR-Vs such as AR-45 can also be found in normal prostate tissue. ${ }^{40} \mathrm{AR}$-Vs are found in men with benign prostate and hormone-naive primary prostate cancer, ${ }^{24}$ but they are mainly observed in those with CRPC, who have higher levels than men with hormone-naïve PC and bone metastases. ${ }^{41}$

The availability of a specific antibody means that AR-V7 is currently the most widely studied AR-V, although there are some published data concerning ARv567es, which was detected in $43 \%$ of the metastases of a series of patients who died of mCRPC. ${ }^{24}$

AR-V7 may be quickly induced by castration or anti-androgen exposure in $\mathrm{VCaP}$ cells and $\mathrm{LNCaP}$ sublines, ${ }^{33,36}$ and its expression is 20 times higher in mCRPC than in hormone-sensitive prostate cancer, ${ }^{38}$ thus leading to a lethal mitotic phenotype. High levels of AR-V7 in metastatic tissues from prostate cancer have been related to faster disease progression and biochemical recurrence, and shorter cancer-specific survival, than low levels. ${ }^{35,42}$ In particular, it has been reported that its expression is significantly up-regulated during prostate cancer progression, and that high AR-V7 levels in primary prostate tumours is correlated with a worse outcome after radical prostatectomy, and that the increase in prostate-specific antigen (PSA) levels is faster in patients with high AR-V7 levels at the time of diagnosis $(\mathrm{P}<0.001) .{ }^{38}$

Qu et al. evaluated AR-V7 expression at different stages of prostate cancer using immunohistochemistry and specific antibody, and clearly showed that it was an independent predictor of the development of CRPC [hazard ratio (HR) 2.62, $\mathrm{P}=0.001$ ], and cancer-specific survival after the transurethral resection of the prostate (TURP) (HR 2.24, $\mathrm{P}=0.033) .^{42}$ In particular, they found that newly diagnosed metastatic patients with AR-V7 expression experienced a shorter median time to CRPC than those without (15 vs 30 months; $\mathrm{P}<0.001$ ), thus suggesting that AR-V7 expression may predict the development of CRPC; that patients with higher levels of AR-V7 expression experienced shorter median cancer survival after TURP than those with lower levels (14 vs 21 months; $\mathrm{P}=0.003)$; and that the expression of $\mathrm{AR}-\mathrm{V} 7$ in patients with newly diagnosed metastatic prostate cancer or CRPC inversely correlated with serum PSA levels $(\mathrm{P}=0.014$ and $\mathrm{P}=0.045)$.

The study of Hornberg also found that metastases arising from prostate cancer in patients with higher AR-V7 expression levels correlated with significantly lower PSA levels, ${ }^{41}$ thus suggesting that androgen-deprivation therapy increases the expression of AR-V7 and inhibits PSA production.

\section{Role of androgen receptors variants in resist- ance to prostate cancer treatments}

About $20-40 \%$ of the patients who receive abiraterone or enzalutamide for the treatment of metastatic CRPC show no PSA response, a clinical condition known as primary resistance..$^{11,13}$ Moreover, patients who initially respond to enzalutamide or abiraterone develop secondary resistance over time. The presence of AR-Vs may be one of the causes of the failure of these new drugs as the expression of a number of ARVs has been observed in xenograft-based models developing resistance to abiraterone, ${ }^{43}$ and high AR-V levels are found in prostate cancer cell lines resistant to enzalutamide. ${ }^{30}$

Since the earliest pre-clinical studies, AR-Vs have been investigated in CTCs taken from peripheral blood samples of patients with metastatic CRPC and, using CTCs, Antonarakis first showed that the lack of the LBD in AR-V7 may be associated with resistance to both enzalutamide and abiraterone ${ }^{15}$ as $39 \%$ of 31 patients treated with enzalutamide and $19 \%$ of 31 patients treated abiraterone showed the expression of AR-V7. They also examined the associations between a AR-V7 status and clinical outcomes, including the PSA response rate, PSA progression-free survival (PFS), clinical or radiographic PFS and overall survival (OS), and found that AR-V7-positive patients had lower PSA response rates 
and experienced shorter PSA, clinical or radiographic PFS and OS than AR-V7-negative patients regardless of whether they had been treated with abiraterone or enzalutamide. None of the men harbouring AR-V7 showed a PSA response as against 53\% of the AR-V7-negative patients in the enzalutamide-treated group and $68 \%$ in the abiraterone-treated group (both $\mathrm{P}=0.004$ ), thus suggesting that $\mathrm{AR}-\mathrm{V} 7$ may play a role as a biomarker in advanced CRPC, with $100 \%$ specificity in predicting the absence of a response to abiraterone or enzalutamide. On the contrary, $53 \%$ of the patients who were AR-V7 negative responded to therapy. Moreover, an exploratory analysis showed that the effect of AR-V7 on these outcomes remained significant in the combined population of all 62 patients and, although the detection of AR-V7 was associated with an increased expression of AR-FL, the prognostic value of AR-V7 remained even after adjusting for AR-FL levels. Among the 42 AR-V7negative patients at baseline (pre-treatment) for whom at least one follow-up sample was available, six became AR-V7-positive status during treatment, whereas all of the AR-V7-positive patients continued to show CTC AR-V7 expression. These findings suggest that any androgen deprivation therapy can increase CTC AR-V7 expression, which may explain the development of secondary resistance to new-generation hormonal therapies. Finally, Antonorakis observed good concordance between the AR-V7 status of CTCs and metastatic tumour tissue, although the small sample size means that further validation is necessary. As the AR-V7/AR-FL ratio tends to be larger in CRPC tissues than hormone-sensitive metastases, ${ }^{41}$ AR-V7 expression often correlates with high AR-FL levels but, although the expression of AR-V7 is lower than that of AR-FLs, it is sufficient to cause a shift to transcriptional signalling mediated by AR-V7 when AR-FL is inhibited by AR-targetingagents despite the absence of androgens.

The potential effect of AR-Vs on chemotherapeutic drug activity is also being investigated. The first data were published by ThadaniMulero, ${ }^{44}$ who found that the type of AR-Vs expressed in vitro and in vivo models affected sensitivity to taxanes. Unlike ARv567, ARv7 (which lacks the hinge region) does not engage the microtubule dynein motor system and, consequently, docetaxel retains its efficacy in the case of ARv567-expressing LuCap86.2 tumour xenografts but not in the case of ARv7-expressing LuCap23.1 tumour xenografts. Other findings suggest that both ARV7 and Arv567es do not depend on the microtubule pathway: in the LNCaP95 cell line, the cells expressing ARV7- and ARV567es show less microtubule binding resistance than the cells without AR-Vs, and it has also been postulated that the presence of AR-Vs can interfere with the interaction between AR-FLs and microtubules, thus reducing the effect of taxanes on the cytoplasmic accumulation of AR-FLs. ${ }^{45}$

Antonorakis et al. have recently assessed the possible relationship between AR-V7 expression in the CTCs of men with MCRPC and the activity of taxanes by evaluating 36 patients treated with docetaxel or cabazitaxel, and found that AR-V7 status was not related to primary resistance to taxane chemotherapy. ${ }^{16}$ They also incorporated updated data from a previous study of 62 patients treated with enzalutamide or abiraterone with the aim of investigating the interaction between ARV7 positivity or negativity and the type of treatment (taxanes $v s$ enzalutamide or abiraterone), and found that the response to taxanes, enzalutamide or abiraterone and their related clinical outcomes seemed to be comparable in AR-V7-negative patients, whereas taxane-based therapy seemed to be more efficacious than enzalutamide or abiraterone in AR-V7-positive patients. In particular, the AR-V7-positive patients treated with taxanes achieved a PSA response more frequently than those treated with enzalutamide- or abiraterone $(41 \%$ vs $0 \% ; \mathrm{P}<0.001)$, and their biochemical PFS $[\mathrm{P}=0.001 ; \mathrm{HR}, 0.19,95 \%$ confidence interval (CI) 0.07-0.52] and PFS ( $\mathrm{P}=0.003$; HR $0.21,95 \%$ CI 0.07-0.59) were significantly longer.

These findings suggesting that the activity of the taxanes is not affected by AR-V7 positivity were recently confirmed by data from a small series of patients treated with cabazitaxel. ${ }^{46}$ In this series, $55 \%$ of 29 patients with $\geq 10$ CTCs were AR-V7 positive, and the frequency of AR-V7 positivity was significantly greater in the patients pre-treated with abiraterone $(5 / 5,100 \%$ vs $7 / 20,35 \% ; \mathrm{P}=0.009)$. AR-V7 positivity was not associated with PFS (HR 0.8; 95\% CI 0.4-1.8) or OS (HR 1.6; 95\% CI 0.6-4.4).

It has also been found that some initially AR-V7-positive patients become AR-V7 negative during taxane therapy, ${ }^{47}$ which suggest decreased pressure on AR-axis signalling by taxanes and therefore potential re-sensitisation to AR-targeted treatment, although the change may be related to the absence of CTCs as a result of the effectiveness of the treatment rather than the down-regulation of AR-V7.

Taken together, the data concerning the relationship between AR-V7 positivity and the therapeutic activity of the new agents confirms the central role of AR machinery in mCRPC. Enzalutamide primarily acts by inhibiting the binding of androgens to the LBD, ${ }^{48}$ but AR-V7 can translocate into the nucleus and regulate gene transcription without ligand binding. Although the main therapeutic effect of abiraterone is due to CYP17 inhibition blocking androgen production, ${ }^{49}$ it has recently been demonstrated that its metabolites directly bind ARs, ${ }^{50}$ thus providing further evidence of possible cross-resistance with enzalutamide. The anti-tumoral activity of the taxanes in MCRPC patients is due to microtubule inhibition, which blocks the nuclear translocation of ligand/receptor complexes ${ }^{51}$ and mitotic fuse stabilization: ${ }^{52}$ the presence of AR-V7 makes the first mechanism ineffective but does not affect the second, thus explaining the retained anti-tumoral activity of the taxanes regardless of the formation of microtubules.

A number of new AR-targeting agents are currently being developed whose mechanisms of action could block AR-V activity. For example, EPI-001, a small molecule, sintokamide peptides, that acts on the NTD of ARs, can block the activity of a constitutively active deletion mutant, AR1-653, that contains the NTD, DBD and hinge region, but not the LBD, ${ }^{53}$ and galeterone, which hits the AR axis with three different mechanisms (CYP17A1 inhibition, ligand-binding AR blockade, and AR degradation), seems to increase AR-V degradation. ${ }^{54}$

\section{Conclusions}

In this era of personalised medicine, the development and validation of prognostic and predictive biomarkers is one of the most widely investigated areas of prostate cancer research, ${ }^{55}$ and has led to the finding of various promising classes of biomarkers, such as kallikreinrelated peptidases, miRNAs, PCA3, and AR mutations. ${ }^{56}$ The clinical use of AR-V7 when selecting the treatment of mCRPC patients could represent a huge step forward in the tailored treatment of prostate cancer, as it has been in the treatment of other tumours such as lung, breast or colorectal cancers.

The available data seem to suggest that it would be better for AR-V7positive men to receive taxane-based chemotherapy than AR-targeted agents, whereas it could be reasonable to treat AR-V7-negative patients with any hormonal or chemotherapy. In a recently published study of a small cohort of patients based on the simultaneous detection of AR-V7 and AR point mutations in CTCs, it was estimated that AR status had a positive predictive value for response and non-response to therapy of $\sim 94 \%$; in addition the authors calculated an effect size for molecularly driven therapy switches for prospective clinical trial planning of $\sim 27 \% .57$

It is worth noting that AR-V7 expression may change during the course of cancer progression and treatment, thus leading to new and intriguing possibilities concerning the systematic use of liquid biopsies to select treatments and test their efficacy.

The role of AR-V7 as a biomarker and the assay used to detect need 
to be prospectively validated in larger clinical trials before they can be recommended in clinical practice. Samples should be uniformly collected, processed and shipped, and the assay should be performed in certified laboratories, standardised and validated: i.e., both analytical and clinical validation are necessary.

Prospective biomarker-stratified clinical trials should be conducted in order to confirm that AR-V7- positive patients have better outcomes with chemotherapy, and that chemotherapy and hormonal therapy may both be considered in AR-V7-negative patients. In other words, patients should be tested for AR-V7 at baseline, and randomised to AR-targeted treatment or taxane chemotherapy. An example of such a study is PRIMCAB (NCT02379390), a multicentric phase II trial of abiraterone or enzalutamide $v s$ cabazitaxel in men with primary resistance to previous enzalutamide or abiraterone treatment.

In conclusion, AR-V7 can be considered a promising biomarker when selecting treatment for mCRPC, but it needs to be analytically and clinically validated before being used in clinical practice to guide therapeutic decision making and promote the development of new targeted drugs.

\section{References}

1. Chan SC, Li Y, Dehm SM. Androgen receptor splice variants activate androgen receptor target genes and support aberrant prostate cancer cell growth independent of canonical androgen receptor nuclear localization signal. J Biol Chem 2012;287:19736-49.

2. Huggins C, Hodges C. Studies on prostatic cancer, I: the effect of castration, of estrogen and of androgen injection on serum phosphatases in metastatic carcinoma of the prostate. Cancer Res 1941;1:293-7.

3. Heidenreich A, Bastian PJ, Bellmunt J, et al. EAU guidelines on prostate cancer. Part II: treatment of advanced, relapsing, and castration-resistant prostate cancer. Eur Urol 2014;65:467-79.

4. Feldman BJ, Feldman D. The development of androgen-independent prostate cancer. Nat Rev Cancer 2001;1:34-45.

5. Marques RB, Dits NF, Erkens-Schulze S, et al. Bypass mechanisms of the androgen receptor pathway in therapy-resistant prostate cancer cell models. PLoS One 2010;5:e13500.

6. Scher HI, Sawyers CL. Biology of progressive, castration-resistant prostate cancer: directed therapies targeting the androgen-receptor signaling axis. J Clin Oncol 2005;23:8253-61.

7. Visakorpi T, Hyytinen E, Koivisto P, et al. In vivo amplification of the androgen receptor gene and progression of human prostate cancer. Nat Genet 1995;9:401-6.

8. Heemers HV, Schmidt LJ, Kidd E, et al. Differential regulation of steroid nuclear receptor coregulator expression between normal and neoplastic prostate epithelial cells. Prostate 2010;70:959-70.

9. Locke JA, Guns ES, Lubik AA, et al. Androgen levels increase by intratumoral de novo steroidogenesis during progression of castration-resistant prostate cancer. Cancer Res 2008;68:6407-15.

10. Sadar MD. Androgen-independent induction of prostate-specific antigen gene expression via cross-talk between the androgen receptor and protein kinase - A signal transduction pathways. J Biol Chem 1999;274:7777-83.

11. Fizazi K, Scher HI, Molina A, et al. Abiraterone acetate for treatment of metastatic castration-resistant prostate cancer: final overall survival analysis of the COU-AA-301 randomised, double-blind, placebo-controlled phase 3 study. Lancet Oncol 2012;13:983-92.

12. Ryan CJ, Smith MR, de Bono JS, et al. Abiraterone in metastatic prostate cancer without previous chemotherapy. N Engl J Med 2013;368:138-48.

13. Scher HI, Fizazi K, Saad F, et al. Increased survival with enzalu- tamide in prostate cancer after chemotherapy. N Engl J Med 2012;367:1187-97.

14. Beer TM, Armstrong AJ, Rathkopf DE, et al. Enzalutamide in metastatic prostate cancer before chemotherapy. N Engl J Med 2014;371:424-33.

15. Antonarakis ES, Lu C, Wang $\mathrm{H}$, et al. AR-V7 and resistance to enzalutamide and abiraterone in prostate cancer. $\mathrm{N}$ Engl $\mathrm{J}$ Med 2014;371:1028-38.

16. Antonarakis ES, Lu C, Luber B, et al. Androgen receptor splice variant 7 and efficacy of taxane chemotherapy in patients with metastatic castration-resistant prostate cancer. JAMA Oncol 2015;1:582-91.

17. Evans RM. The steroid and thyroid hormone receptor superfamily. Science 1988;240:889-95.

18. Bain DL, Heneghan AF, Connaghan-Jones KD, Miura MT. Nuclear receptor structure: implications for function. Annu Rev Physiol 2007;69:201-20.

19. Jenster G, van der Korput HA, van Vroonhoven C, et al. Domains of the human androgen receptor involved in steroid binding, transcriptional activation, and subcellular localization. Mol Endocrinol 1991;5:1396-404.

20. Heemers HV, Tindall DJ. Androgen receptor (AR) coregulators: a diversity of functions converging on and regulating the AR transcriptional complex. Endocr Rev 2007;28:778-808.

21. Tepper CG, Boucher DL, Ryan PE, et al. Characterization of a novel androgen receptor mutation in a relapsed CWR22 prostate cancer xenograft and cell line. Cancer Res 2002;62:6606-14.

22. Dehm SM, Tindall DJ. Alternatively spliced androgen receptor variants. Endocr Relat Cancer 2001;18:R183-96.

23. Xu D, Zhan Y, Qi Y, et al. Androgen receptor splice variants dimerize to transactivate target genes. Cancer Res 2015;75:3663-71.

24. Sun S, Sprenger CC, Vessella RL, et al. Castration resistance in human prostate cancer is conferred by a frequently occurring androgen receptor splice variant. J Clin Invest 2010;120:2715-30.

25. Cao B, Qi Y, Zhang G, et al. Androgen receptor splice variants activating the full-length receptor in mediating resistance to androgen-directed therapy. Oncotarget 2014;5:1646-56.

26. An J, Wang C, Deng Y, et al. Destruction of full-length androgen receptor by wild-type SPOP, but not prostate-cancer-associated mutants. Cell Rep 2014;6:657-69.

27. McGrath MJ, Binge LC, Sriratana A, et al. Regulation of the transcriptional coactivator FHL2 licenses activation of the androgen receptor in castrate-resistant prostate cancer. Cancer Res 2013;73:5066-79.

28. Peacock S0, Fahrenholtz CD, Burnstein KL. Vav3 enhances androgen receptor splice variant activity and is critical for castrationresistant prostate cancer growth and survival. Mol Endocrinol 2012; 26:1967-79.

29. Mediwala SN, Sun H, Szafran AT, et al. The activity of the androgen receptor variant AR-V7 is regulated by FOX01 in a PTEN-PI3K-AKTdependent way. Prostate 2013;73:267-77.

30. Li Y, Chan SC, Brand LJ, et al. Androgen receptor splice variants mediate enzalutamide resistance in castration-resistant prostate cancer cell lines. Cancer Res 2013;73:483-9.

31. Nyquist MD, Dehm SM. Interplay between genomic alterations and androgen receptor signaling during prostate cancer development and progression. Horm Cancer 2013;4:61-9.

32. Li Y, Alsagabi M, Fan D, et al. Intragenic rearrangement and altered RNA splicing of the androgen receptor in a cell-based model of prostate cancer progression. Cancer Res 2011;71:2108-17.

33. Liu LL, Xie N, Sun S, et al. Mechanisms of the androgen receptor splicing in prostate cancer cells. Oncogene 2014;33:3140-50.

34. Jernberg E, Thysell E, Bovinder YE, et al. Characterization of prostate cancer bone metastases according to expression levels of 
steroidogenic enzymes and androgen receptor splice variants. PLoS One 2013;8:e77407.

35. Guo Z, Yang X, Sun F, et al. A novel androgen receptor splice variant is up-regulated during prostate cancer progression and promotes androgen depletion-resistant growth. Cancer Res 2009;69:2305-13.

36. Hu R, Lu C, Mostaghel EA, et al. Distinct transcriptional programs mediated by the ligand-dependent full-length androgen receptor and its splice variants in castration-resistant prostate cancer. Cancer Res 2012;72:3457-62.

37. Zhang X, Morrissey C, Sun S, et al. Androgen receptor variants occur frequently in castration resistant prostate cancer metastases. PLoS One 2011;6:e27970.

38. Hu R, Dunn TA, Wei S, et al. Ligand-independent androgen receptor variants derived from splicing of cryptic exons signify hormonerefractory prostate cancer. Cancer Res 2009;69:16-22.

39. Lu C, Luo J. Decoding the androgen receptor splice variants. Transl Androl Urol 2013;2:178-86.

40. Ahrens-Fath I, Politz 0, Geserick C, Haendler B. Androgen receptor function is modulated by the tissue-specific AR45 variant. FEBS J 2005;272:74-84.

41. Hornberg E, Ylitalo EB, Crnalic S, et al. Expression of androgen receptor splice variants in prostate cancer bone metastases is associated with castration-resistance and short survival. PLoS One 2001;6:e19059.

42. Qu Y, Dai B, Ye D, et al. Constitutively active AR-V7 plays an essential role in the development and progression of castration-resistant prostate cancer. Sci Rep 2015;5:7654.

43. Mostaghel EA, Marck BT, Plymate SR, et al. Resistance to CYP17A1 inhibition with abiraterone in castration-resistant prostate cancer: induction of steroidogenesis and androgen receptor splice variants. Clin Cancer Res 2011;17:5913-25.

44. Thadani-Mulero M, Portella L, Sun S, et al. Androgen receptor splice variants determine taxane sensitivity in prostate cancer. Cancer Res 2014;74:2270-82.

45. Zhang G, Liu X, Li J, et al. Androgen receptor splice variants circumvent AR blockade by microtubule-targeting agents. Oncotarget 2015;6:23358-71.

46. Onstenk W, Sieuwerts AM, Kraan J, et al. Efficacy of cabazitaxel in castration-resistant prostate cancer is independent of the presence of ar-v7 in circulating tumor cells. Eur Urol 2015;68:939-45.

47. Nakazawa M, Lu C, Chen Y, et al. Serial blood-based analysis of ARV7 in men with advanced prostate cancer. Ann Oncol 2015;26:185965.

48. Tran C, Ouk S, Clegg NJ, et al. Development of a second-generation antiandrogen for treatment of advanced prostate cancer. Science 2009;324:787-90.

49. Jarman M, Barrie SE, Llera JM. The 16,17-double bond is needed for irreversible inhibition of human cytochrome p45017alpha by abiraterone (17-(3-pyridyl)androsta-5, 16-dien-3beta-ol) and related steroidal inhibitors. J Med Chem 1998;41:5375-81.

50. Li Z, Bishop AC, Alyamani M, et al. Conversion of abiraterone to D4A drives anti-tumour activity in prostate cancer. Nature 2015;523:347-51.

51. Darshan MS, Loftus MS, Thadani-Mulero M, et al. Taxane-induced blockade to nuclear accumulation of the androgen receptor predicts clinical responses in metastatic prostate cancer. Cancer Res 2011;71:6019-29.

52. Altmann KH. Microtubule-stabilizing agents: a growing class of important anticancer drugs. Curr Opin Chem Biol 2001;5:424-31.

53. Sadar MD, Williams DE, Mawji NR, et al. Sintokamides A to E, chlorinated peptides from the sponge Dysidea sp. that inhibit transactivation of the $\mathrm{N}$-terminus of the androgen receptor in prostate cancer cells. Org Lett 2008;10:4947-50.

54. Njar VC, Brodie AM. Discovery and development of Galeterone (TOK-001 or VN/124-1) for the treatment of all stages of prostate cancer. J Med Chem 2015;58:2077-87.

55. Spahn M, Boxler S, Joniau S, et al. What is the need for prostatic biomarkers in prostate cancer management? Curr Urol Rep 2015;16:70.

56. Kontos CK, Adamopoulos PG, Scorilas A. Prognostic and predictive biomarkers in prostate cancer. Expert Rev Mol Diagn 2015;15:1567-76.

57. Steinestel J, Luedeke M, Arndt A, et al. Detecting predictive androgen receptor modifications in circulating prostate cancer cells. Oncotarget 2015 [Epub ahead of print] 